\title{
ANALYTICAL SOLUTIONS TO BOUNDARY VALUE PROBLEM OF FREE VIBRATION OF SANDWICH THIN CIRCULAR PLATES WITH DISCRETE ELEMENTS
}

\author{
K.K. ŻUR* \\ Business Informatics and Logistics \\ Faculty of Management \\ Bialystok University of Technology \\ Ojca Tarasiuka street 2, 16-001 Kleosin, POLAND \\ E-mail: k.zur@pb.edu.pl \\ J. JAROSZEWICZ and $Ł$. DRAGUN \\ Production Management \\ Faculty of Management \\ Bialystok University of Technology \\ Ojca Tarasiuka street 2, 16-001 Kleosin, POLAND \\ E-mails: j.jaroszewicz@pb.edu.pl; 1.dragun@pb.edu.pl
}

\begin{abstract}
In the paper the influence function and the method of partial discretization in free axisymmetric vibration analysis of multilayered circular plates of constant and linearly variable thickness were presented. The effects of shear deformation and rotary inertia for the core as well as the facings were neglected. An analytical investigation based on the classical plate theory was made for the multilayered plate which satisfies Sokołowski's condition. Discretization of mass and replacing stiffness of a fixed circular plate were presented. Formulas of influence matrix and Bernstein-Kieropian's estimators for different steps of discretization were defined. The influence of variable distribution of parameters on the value of double estimators of natural basic and higher frequency of a sandwich circular plate was investigated.
\end{abstract}

Key words: free vibrations, sandwich circular plate, non-homogeneous material, variable parameters, annular mass.

\section{Introduction}

A sandwich plate is an important structural element in aeronautical, astronautical and naval engineering. This plate is composed of three layers, a thick core and two thin faces. A sandwich construction has been used for many years in aerospace industries and aviation as well as in marine and civil engineering applications due to the specific stiffness, light weight and design versatility besides good damping characteristics and maximum fatigue resistance.

A lot of researchers have been concerned with the dynamic behavior of sandwich structures (Magnucki et al., 2014; Lal and Rani, 2013; Zhang, 2013; Duan, 2005; Kączkowski, 2000). The boundary value problem of transverse vibrations for the multilayered and homogeneous fixed plates is one of the most frequently considered issues in engineering practice (Starovoitov et al., 2009; Ebrahimi and Rastgo, 2008). The necessity of considering the discrete masses and variable thickness of the plate leads to particular problems. Many authors showed that ignoring varying thickness of the plate, even in the case of thin plates, leads to significant errors in the calculation of natural frequencies (Conway, 1958b). Conway (1958b) found characteristic equations using Bessel functions for the particular case where the thickness of the plate varies

\footnotetext{
* To whom correspondence should be addressed
} 
as a power function. The exact solution to the vibration problems for a circular and annular constant and variable thickness plates having an additional annular mass and spring inclusions was provided by among others, Roberson (1951), Szewczyk (2007) and Kukla (2007).

In this paper free axisymmetric vibrations of a sandwich circular plate of constant and linearly variable thickness with additional annular mass were considered. For the vibrations of the plate with variable parameters the method of partial discretization has been used. It is based on the properties of the influence function used to, e.g., the analysis of the bending curve and reactions of elastic supports of a beam considered in an earlier work (Jaroszewicz et al., 2014).

\section{Formulation of the boundary value problem}

If we consider a three-layered circular plate which satisfies Sokołowski's condition (Sokołowski, 1958; Kączkowski, 2000) presented in the following form

$$
\begin{aligned}
& \frac{E_{f}}{E_{c}}=n^{2}, \\
& n h \leq h_{H}
\end{aligned}
$$

where $E_{f}$ - modulus of elasticity of facing, $E_{c}$ - modulus of elasticity of core, $h$ - thickness of three-layered plates, $h_{H}$-thickness of homogeneous plates, then the plate will satisfy the classical plate theory (CPT).

The equation of free axisymmetric vibrations of a circular plate of variable thickness (CPT) has the form (Timoshenko and Woinowsky-Krieger, 1940)

$$
D \frac{\partial}{\partial r}\left(\frac{\partial^{2} W}{\partial r^{2}}+\frac{1}{r} \frac{\partial W}{\partial r}\right)+\frac{\partial D}{\partial r}\left(\frac{\partial^{2} W}{\partial r^{2}}+\frac{v}{r} \frac{\partial W}{\partial r}\right)=-\frac{1}{r} \int_{0}^{r} \rho h \frac{\partial^{2} W}{\partial t^{2}} r d r
$$

Taking into consideration $W=w(r) e^{i \omega t}$ and $D=D_{0} r^{m}\left(D_{0}=\frac{E h^{3}}{12\left(1-v^{2}\right)} ; 0 \leq m<6 ; m=v^{-1}\right)$, Eq.(2.2) has the following form (Conway, 1958b)

$$
\begin{aligned}
& r^{4} \frac{d^{4} w}{d r^{4}}+(2 m+2) r^{3} \frac{d^{3} w}{d r^{3}}+\left(m+v m-1+m^{2}\right) r^{2} \frac{d^{2} w}{d r^{2}}+ \\
& +\left(v m^{2}-v m-m+1\right) r \frac{d w}{d r}-\frac{12 \rho \omega^{2}\left(1-v^{2}\right)}{E h^{2}} r^{4-\frac{2}{3} m} w=0
\end{aligned}
$$

where $r$ - radial coordinate $(0<r \leq R), v$ - Poisson's ratio, $E$ - modulus of elasticity, $m$ - coefficient of variable thickness of the plate, $h$ - thickness of the plate, $\rho$ - density, $\omega$-parameter of frequency, $w(r)-$ function of deflection of the plate, $D$ - stiffness bending of the plate of variable thickness.

The equation of axisymmetric vibrations of sandwich circular plates with spring and mass inclusions has the following form (Jaroszewicz and Zoryj, 2005)

$$
L[w]+\frac{M}{D_{z}} \omega^{2} w-\sum_{i=1}^{K} \alpha_{i} w\left(r_{i}\right) \delta\left(r-r_{i}\right)=0
$$




$$
\begin{aligned}
& L[w] \equiv \frac{d^{4} w}{d r^{4}}+\frac{2}{r}(m+1) \frac{d^{3} w}{d r^{3}}+\frac{1}{r^{2}}\left(m+v m-1+m^{2}\right) \frac{d^{2} w}{d r^{2}}+ \\
& +\left(v m^{2}-v m-m+1\right) \frac{1}{r^{3}} \frac{d w}{d r} \\
& \alpha_{i}=D_{z}^{-1}\left(m_{i} \omega^{2}-c_{i}\right), \quad(i \in<1 \div K>)
\end{aligned}
$$

where $L[w]$ - differential operator of Euler's equation appropriately for a plate of constant and linearly variable thickness, $D_{z}$ - replacing stiffness of the sandwich plate, $M$ - mass of the plate, $\alpha_{i}$-inclusions, $m_{i}$ - annular mass on radius $r_{i}, c_{i}$ - stiffness of elastic supports on radius $r_{i}$ (for considered plates $c_{i}=0$ ), $K-$ number of inclusions, $\delta$ - Dirac's delta.

In a particular case, operator (2.5) for the plate of constant thickness is in the following form

$$
L[w]_{m=0} \equiv \frac{d^{4} w}{d r^{4}}+\frac{2}{r} \frac{d^{3} w}{d r^{3}}+\frac{1}{r^{2}} \frac{d^{2} w}{d r^{2}}+\frac{1}{r^{3}} \frac{d w}{d r}
$$

The differential operator (2.5) for the plate of linearly variable thickness has the form

$$
L[w]_{m=3} \equiv \frac{d^{4} w}{d r^{4}}+\frac{8}{r} \frac{d^{3} w}{d r^{3}}+\frac{12}{r^{2}} \frac{d^{2} w}{d r^{2}}
$$

Fixed circular plates have boundary conditions in the following form (Vasylenko and Oleksiejčuk, 2004)

$$
w(R)=0, \quad \theta(R)=\frac{d w}{d r}(R)=0, \quad w(0) \leq h / 2, \quad \theta(0)=\frac{d w}{d r}(0)=0
$$

where $w(0)$ is an admissible bending which satisfies the classical plate theory. The solutions to boundary value problem (2.4), (2.9) with their derivatives are limited in $r=0$.

\section{Discretization of mass of sandwich circular plates of constant and linearly variable thickness}

The radius of distribution plate's mass $r_{i}$ has the following form

$$
r_{i}=\frac{R(2 i-1)}{K}, \quad i \in<1, K>
$$

Based on the Guldin-Pappus theorem the equations for $i$-th mass of the three-layered circular plate of constant $(m=0)$ and linearly $(m=0)$ variable thickness were defined in the following form

$$
\frac{m_{i}}{2 \pi}=\aleph_{i} \cdot R^{2} q_{f}\left(h-h_{c}+h_{c} \frac{\rho_{c}}{\rho_{f}}\right),
$$




$$
\aleph_{i}=\frac{1+2(i-1)}{2 K^{2}} \quad \text { for } \quad m=0, \quad \aleph_{i}=\frac{i^{3}-(i-1)^{3}}{3 K^{3}} \quad \text { for } \quad m=3
$$

where $\rho_{f}$ - density of facing, $\rho_{c}$ - density of core, $h$ - thickness of the three-layered plate, $h_{c}-$ thickness of core of the plate.

The sum of the mass from discretization equals the total mass of the plate

$$
\sum_{i=1}^{K} \frac{m_{i}}{2 \pi}=\pi R^{2} \rho_{f}\left(h-h_{c}+h_{c} \frac{\rho_{c}}{\rho_{f}}\right) .
$$

Examples of results of calculation of the coefficient $\aleph_{i}$ for discretization $K \in<1 \div 3>$ were presented in Tab.1.

Table 1. Value $\aleph_{i}$ for discretization $K \in<1 \div 3>$ of mass of the plate of constant and linearly variable thickness.

\begin{tabular}{|c|c|c|}
\hline \multicolumn{2}{|c|}{$\begin{array}{c}\text { Circular plate of } \\
\text { constant thickness }\end{array}$} & $\begin{array}{c}\text { Circular plate of } \\
\text { linearly variable thickness }\end{array}$ \\
\hline$K$ & $\aleph_{i}$ & $\aleph_{i}$ \\
\hline 1 & $\aleph_{1}=\frac{1}{2}$ & $\aleph_{1}=\frac{1}{3}$ \\
\hline 2 & $\aleph_{1}=\frac{1}{2 \cdot 4} \quad \aleph_{2}=\frac{3}{2 \cdot 4}$ & $\aleph_{1}=\frac{1}{3 \cdot 8} \quad \aleph_{2}=\frac{7}{3 \cdot 8}$ \\
\hline 3 & $\aleph_{1}=\frac{1}{2 \cdot 9} \quad \aleph_{2}=\frac{3}{2 \cdot 9} \quad \aleph_{3}=\frac{5}{2 \cdot 9}$ & $\aleph_{1}=\frac{1}{3 \cdot 27} \quad \aleph_{2}=\frac{7}{3 \cdot 27} \quad \aleph_{3}=\frac{19}{3 \cdot 27}$ \\
\hline
\end{tabular}

The additional annular mass $m_{0}$ Eq.(2.6) placed on radius $r_{0}$ has the following form

$$
\mu_{0}=\frac{m_{0}}{\pi R^{2} \rho_{f}\left(h-h_{c}+h_{c} \frac{\rho_{c}}{\rho_{f}}\right)}, \quad \chi_{0}=\frac{r_{0}}{R}
$$

The radius of additional mass must satisfy the condition $0<r_{1}<r_{2}<\ldots<r_{K}<R$. The value of additional mass equals $\mu_{0} \leq 0.2$ (Roberson, 1951).

\section{Influence matrix}

An example of calculation of the influence matrix for the circular plate of constant and linearly variable thickness was presented. The limited solution $(r=0)$ of Euler's Eq.(2.5) $L[w]=0$ for the circular plate of constant thickness has the form

$$
w(r)=C_{0}+C_{1} r^{2}-F_{j} \cdot K_{0}\left(r, r_{j}\right) \cdot H\left(r-r_{j}\right)
$$


where: $C_{i}$ - constants of integral, $\mathrm{H}(r)$ - Heaviside's function, $F_{j}$ - unit force distribution on radius $r_{j}, K_{0}\left(r, r_{j}\right)$ - fundamental solution of $L[w]$ which has the form

$$
K_{0}\left(r, r_{j}\right)=\frac{r_{j}}{4}\left[r_{j}^{2}-r^{2}+\left(r^{2}+r_{j}^{2}\right) \ln \frac{r_{j}}{r}\right], \quad 0 \leq r, \quad r_{j} \leq R .
$$

The Cauchy function $K_{0}\left(r, r_{j}\right)$ for the circular plate of constant thickness for normalized $\frac{r}{R}$ and $\frac{r_{j}}{R}$ was presented in Fig.1.

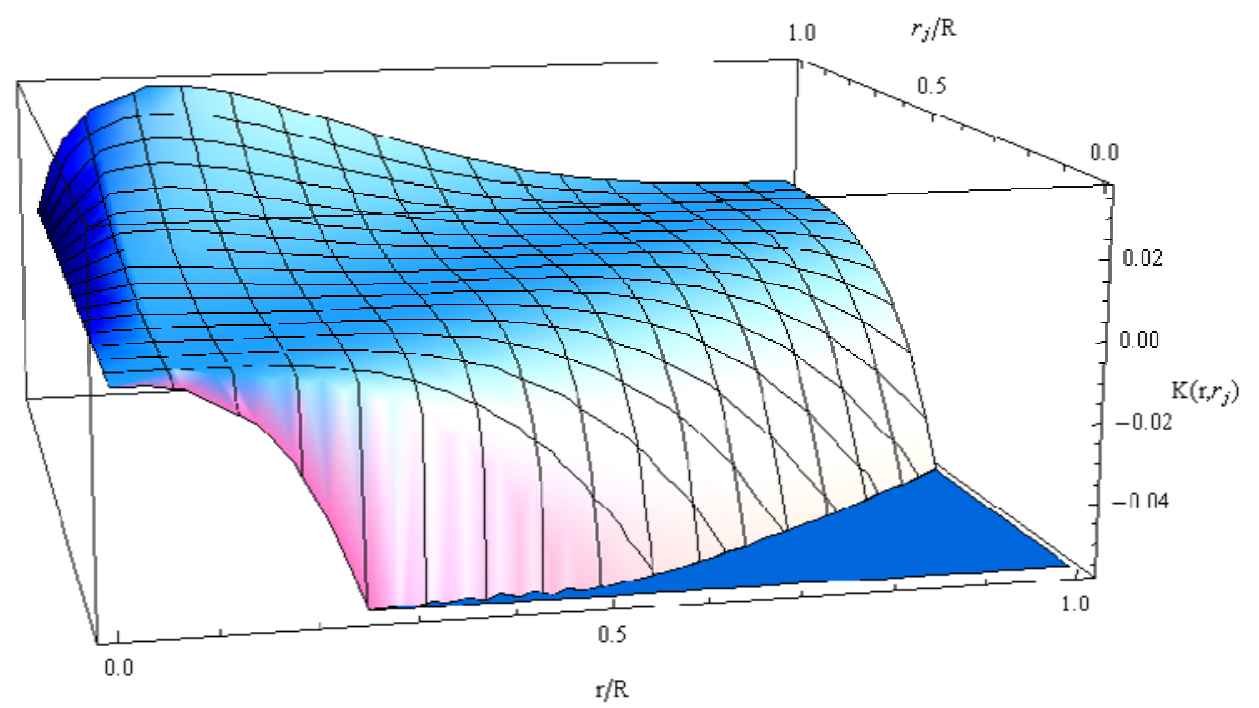

Fig.1. Cauchy function $K_{0}\left(r, r_{j}\right)$ for the circular plate of constant thickness.

The limited solution of Euler's Eq.(4.1) and two first boundary conditions (2.9) were used to calculate constants $C_{0}, C_{1}$. Taking into consideration equations $\beta_{i j}=u_{j}\left(r_{i}\right)$ and $F_{j}=1$, the formula of the influence matrix has the form (Jaroszewicz and Zoryj, 2005)

$$
\beta_{i j}=\frac{1}{2 R}\left(K_{0}^{\prime}\left(R, r_{j}\right) \cdot\left(R^{2}-r_{i}^{2}\right)-2 R \cdot K_{0}\left(R, r_{j}\right)\right) .
$$

After transforms Eq.(4.3) has the following form after transformations

$$
\begin{aligned}
& \beta_{i j}=\frac{R^{2}}{8}\left(1-\frac{r_{j}^{2}-r_{i}^{2}}{R^{2}}-\frac{r_{j}^{2} r_{i}^{2}}{R^{4}}+2 \frac{r_{j}^{2}+r_{i}^{2}}{R^{2}} \ln \frac{r_{j}}{R}\right), \quad i \leq j, \\
& \beta_{i j}=\beta_{j i}, \quad \beta_{i i}=\frac{R^{2}}{8}\left(1-\frac{r_{i}^{4}}{R^{4}}+\frac{4 r_{i}^{2}}{R^{2}} \ln \frac{r_{j}}{R}\right) .
\end{aligned}
$$

For $\chi_{i}=\frac{r_{i}}{R}$ and $\chi_{j}=\frac{r_{j}}{R}$ coefficients of the influence matrix for the plate of constant thickness have the form 


$$
\beta_{i j}=\frac{R^{2}}{8}\left(1+\chi_{i}^{2}-\chi_{j}^{2}-\chi_{i}^{2} \chi_{j}^{2}+2\left(\chi_{i}^{2}+\chi_{j}^{2}\right) \ln \chi_{j}\right)
$$

Based on the method of calculation of coefficients of the influence matrix presented above, Cauchy function $K_{0}\left(r, r_{j}\right)$ (Fig.2) and the formula for coefficients of the influence matrix for the circular plate of linearly variable thickness have the following form

$$
\begin{aligned}
& K_{0}\left(r, r_{j}\right)=\frac{1}{6}\left(r_{j} r^{2}-r_{j}^{5} r^{-2}\right)+\frac{1}{2}\left(r_{j}^{4} r^{-1}-r_{j}^{3}\right), \\
& \beta_{i j}=\frac{R^{2}}{3 D_{z} \chi_{i}}\left(\frac{3}{2} \chi_{i}\left(\chi_{i}+\chi_{j}\right)+\frac{3 \chi_{i}}{2 \chi_{j}}-\frac{1}{2}\left(\frac{\chi_{i}}{\chi_{j}}\right)^{2}-3 \chi_{i}-\chi_{i}^{2} \chi_{j}\right) .
\end{aligned}
$$

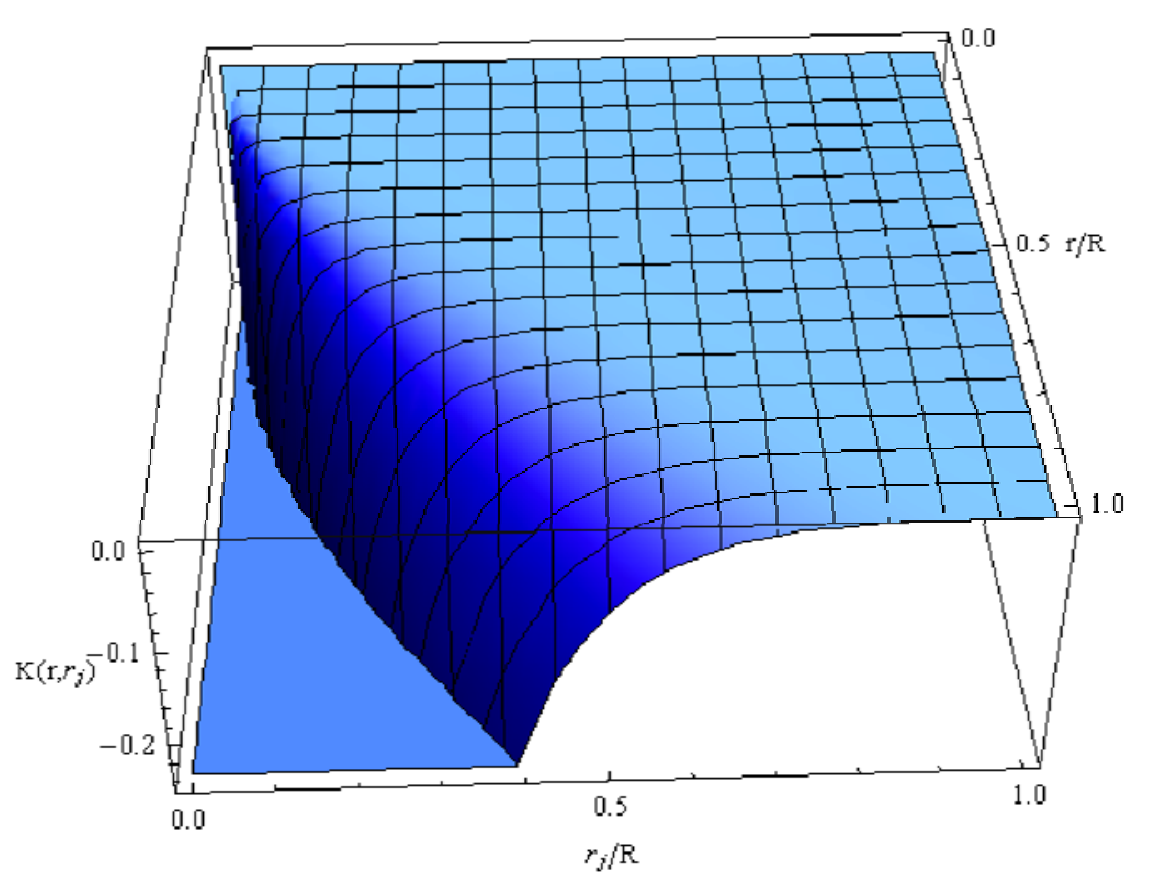

Fig.2. Cauchy function $K_{0}\left(r, r_{j}\right)$ for circular plate of linearly variable thickness.

\section{Characteristic equation of natural frequency of sandwich circular plates}

The record for continuous or discrete- continuous mass distribution systems can be replaced with one, two and $n$-degrees of freedom, which are characterized by the same function of stiffness. The plate's mass was placed on the rings with a certain radius. The total weight of the replacement is equal to the weight of the plate. This procedure is used to get the universal characteristic equation of elastic systems based on inverse equations of motion and the theorem of Betti-Maxwell (Solecki and Szymkiewicz, 1964). The inverse equations of motion $(K=2)$ for the circular plates have the following form

$$
\sum_{j=1}^{2} M_{j} \beta_{i j} \frac{d^{2} q_{j}}{d t^{2}}+q_{i}=0, \quad M_{j}=\frac{m_{j}}{2 \pi}
$$


The obtained characteristic equation has the following form

where

$$
\Delta=a_{0}-a_{1} \lambda+a_{2} \lambda^{2}=0
$$

$$
\begin{aligned}
& a_{0}=1, \quad a_{1}=\sum_{i=1}^{2} \frac{m_{i}}{2 \pi} \beta_{i i}, \quad a_{2}=\frac{m_{1} m_{2}}{4 \pi^{2}}\left(\beta_{11} \beta_{22}-\beta_{12}^{2}\right), \\
& \lambda=\omega^{2} \frac{R^{4} \rho_{f}\left(h-h_{c}+h_{c} \frac{\rho_{c}}{\rho_{f}}\right)}{D_{z}}, \\
& D_{z}=2\left[\int_{0}^{h_{c} / 2} \frac{E_{c} z^{2}}{1-v_{c}^{2}} d z+\int_{h_{c} / 2}^{h / 2} \frac{E_{f} z^{2}}{1-v_{f}^{2}} d z\right]=\frac{1}{12}\left[\frac{E_{c} h_{c}^{3}}{1-v_{c}^{2}}+\frac{E_{f}\left(h^{3}-h_{c}^{3}\right)}{1-v_{f}^{2}}\right]
\end{aligned}
$$

where $v_{c}$ - Poisson's ratio of core, $v_{f}$ - Poisson's ratio of facing.

Taking into consideration an additional annular mass $\frac{m_{0}}{2 \pi}$ and parameters $\left(\mu_{0}, \chi_{0}\right)$ the characteristic equation has a new form

where

$$
\tilde{\Delta}=\widetilde{a_{0}}-\widetilde{a_{1}} \lambda+\widetilde{a_{2}} \lambda^{2}=0
$$

$$
\widetilde{a_{0}}=1 ; \quad \widetilde{a_{1}}=\frac{m_{00}}{2 \pi} \beta_{00}+a_{1} ; \quad \widetilde{a_{2}}=\frac{m_{0} m_{1}}{4 \pi^{2}}\left(\beta_{00} \beta_{11}-\beta_{01}^{2}\right)+\frac{m_{0} m_{2}}{4 \pi^{2}}\left(\beta_{00} \beta_{22}-\beta_{02}^{2}\right)+a_{2}
$$

The coefficients of characteristic Eq.(5.6) for $K>2$ have the following form

$$
\widetilde{a_{0}}=1, \quad \widetilde{a_{1}}=\sum_{i=0}^{K} \frac{m_{i}}{2 \pi} \beta_{i i}, \quad \widetilde{a_{2}}=\sum_{i=0}^{K-1} \sum_{j=i+1}^{K} \frac{m_{i} m_{j}}{4 \pi^{2}}\left|\begin{array}{cc}
\beta_{i i} & \beta_{i j} \\
\beta_{j i} & \beta_{j j}
\end{array}\right| .
$$

The natural basic frequency $\omega_{0}$ of sandwich circular plates can be calculated using BernsteinKieropian's equation (Bernstein and Kieropian, 1960)

$$
\omega_{0}=\gamma_{0} \frac{1}{R^{2}} \sqrt{\frac{D_{z}}{\rho_{f}\left(h-h_{c}+h_{c} \frac{\rho_{c}}{\rho_{f}}\right)}}, \quad \gamma_{0}=\frac{\left(y_{-}\right)+\left(y_{+}\right)}{2}
$$

where estimators of the basic natural frequency have the form

$$
\left(y_{-}\right)=\left(\frac{\widetilde{a_{0}}}{\sqrt{{\widetilde{a_{1}}}^{2}-2 \widetilde{a_{0} a_{2}}}}\right)^{\frac{1}{2}}, \quad\left(y_{+}\right)=\left(\frac{2 \widetilde{a_{0}}}{\widetilde{a_{1}}+\sqrt{{\widetilde{a_{1}}}^{2}-4 \widetilde{a_{0}} \widetilde{a_{2}}}}\right)^{\frac{1}{2}} .
$$




\section{Results of calculation}

If factors of characteristic equation (5.6) are omitted

$$
\begin{array}{ll}
{\left[\frac{m_{i}}{2 \pi}\right] \sim R^{2} \rho_{f}\left(h-h_{c}+h_{c} \frac{\rho_{c}}{\rho_{f}}\right),} & {\left[\beta_{i j}\right] \sim \frac{R^{2}}{D_{z}},} \\
{\left[\widetilde{a_{1}}\right] \sim \frac{R^{4}}{D_{z}} \rho_{f}\left(h-h_{c}+h_{c} \frac{\rho_{c}}{\rho_{f}}\right),} & {\left[\widetilde{a_{2}}\right] \sim\left(\frac{R^{4}}{D_{z}} \rho_{f}\left(h-h_{c}+h_{c} \frac{\rho_{c}}{\rho_{f}}\right)\right)^{2},}
\end{array}
$$

estimators of axisymmetric frequency will be the same for the fixed multilayered and homogeneous circular plates and only the absolute value of frequency will be different. The results of an analytical calculation of estimators of the first three frequency parameters, coefficients of the influence matrix and the coefficients of the characteristic series for circular plate of constant thickness and additional mass $\left(\mu_{0}=0.1, \chi_{0}=\frac{1}{50}\right)$ were presented in Tab.2. The results of calculation of estimators of three axisymmetric frequency parameters for circular plates of linearly variable thickness with additional mass were presented in Tab.3.

Table 2. Results of calculations $(K \in<2 ; 3>)$ of radius $r_{i}$, masses $m_{i}$, influence matrix $[\beta]$ and estimators $\gamma$ for homogeneous and sandwich circular plates of constant thickness.

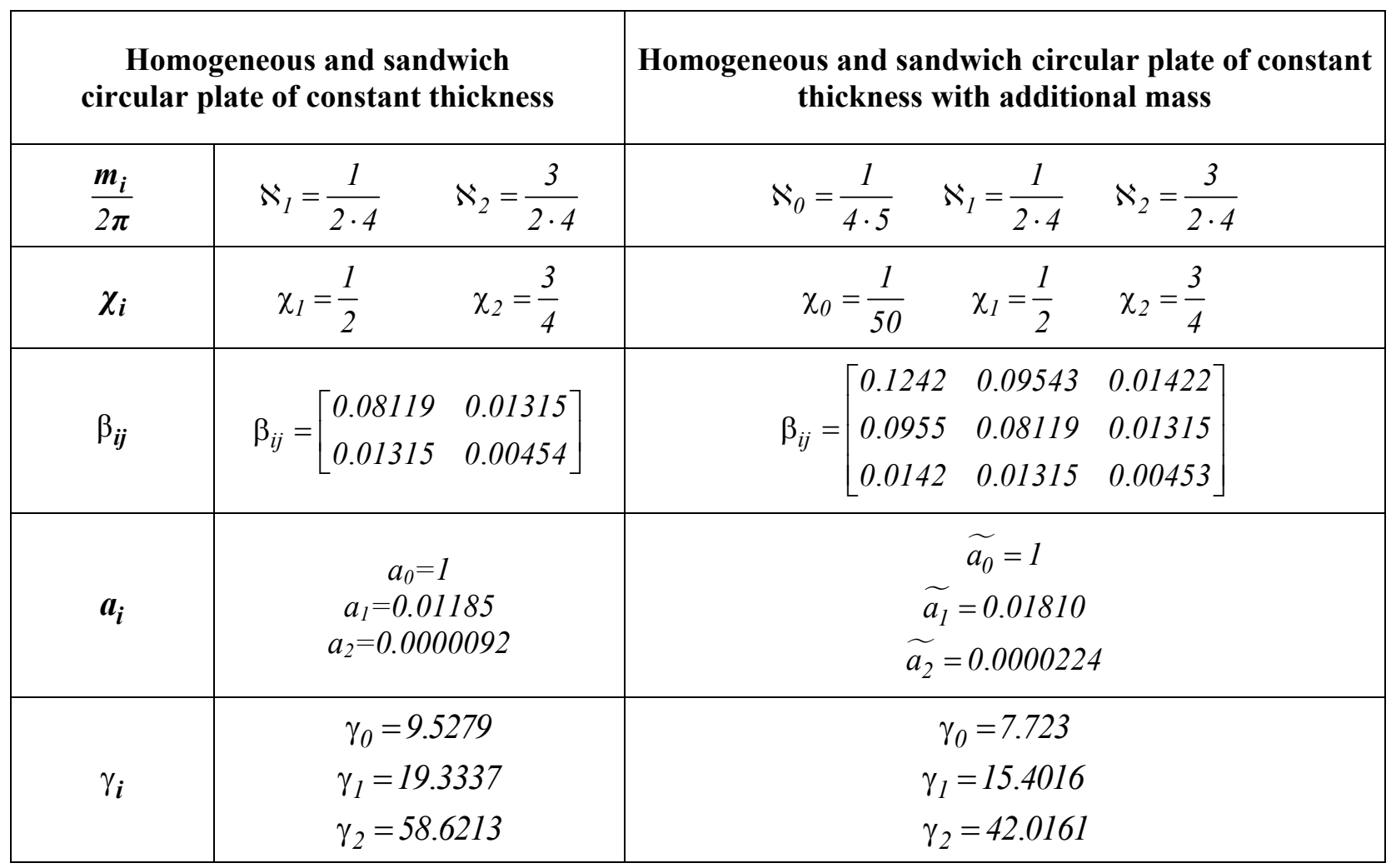


Table 3. Results of calculations $(K \in<2 ; 3>)$ of radius $r_{i}$, masses $m_{i}$, influence matrix [ $\beta$ ] and estimators $\gamma$ for homogeneous and sandwich circular plates of linearly variable thickness.

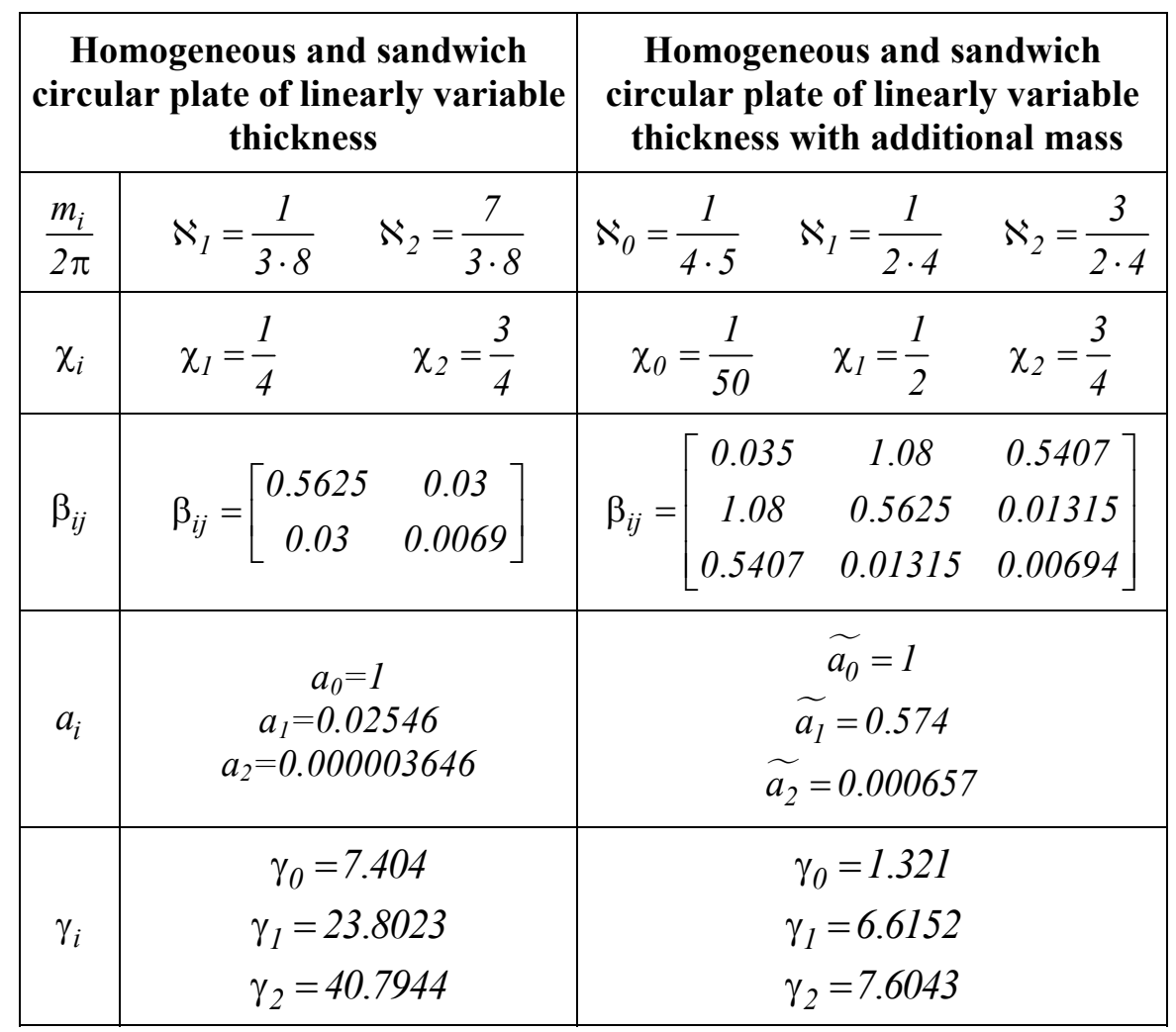

The examples of the influence of step discretization on the values of estimators of the basic frequency $\gamma_{0}$ are presented in Fig.3. The relative error between the exact values $\gamma_{0}$ (Conway, 1958b; Roberson, 1951) and the values from the method of partial discretization are presented in Fig.4.

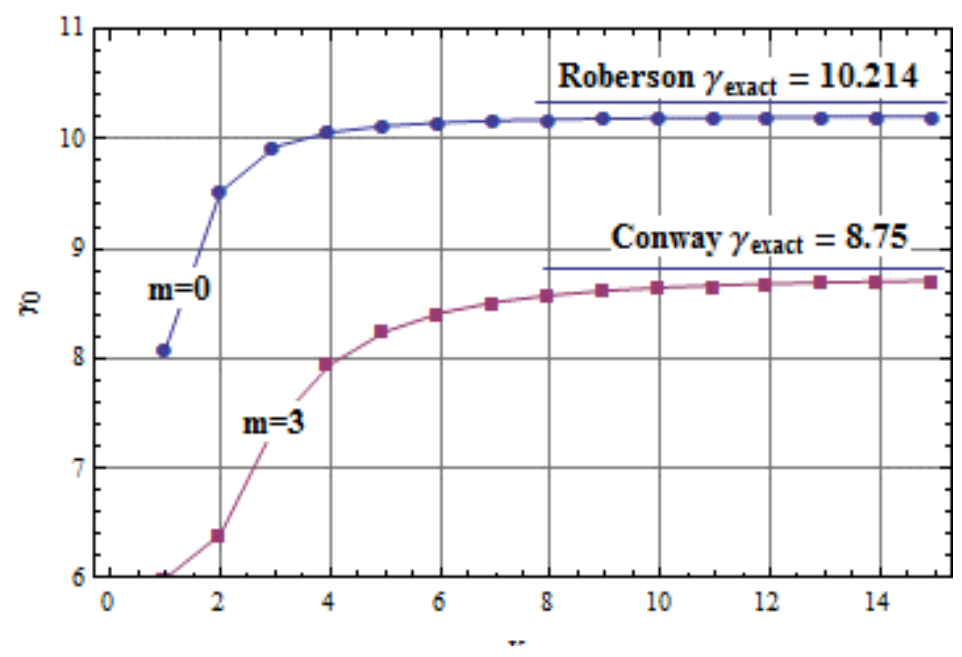

Fig.3. Influence of step discretization on the values of estimators of the basic frequency $\gamma_{0}$ for the plate of constant $(m=0)$ and linearly $(m=3)$ variable thickness. 


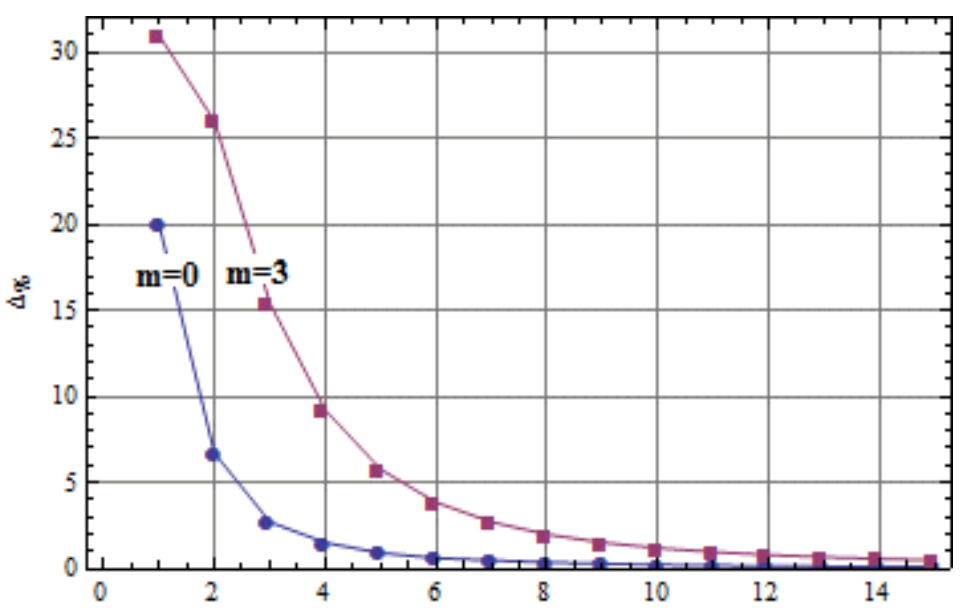

Fig.4. Relative error between the exact values $\gamma_{0}$ and values from the method of partial discretization for the plate of constant $(m=0)$ and linearly $(m=3)$ variable thickness.

\section{Conclusions}

Thin sandwich and homogeneous circular plates of constant and varying thickness carrying concentrated mass have been investigated. The influence of variable thickness and additional annular mass on double estimators of the basic and higher frequency of circular plates were presented. Figures 3 and 4 show that the results produced by the discretization method are close to the exact values for only 15 steps of discretization. Double estimators have the same value for homogeneous and sandwich circular plates of constant thickness and for plates of linearly variable thickness. Non-homogeneous material has no influence on double estimators of the basic and higher frequency. Important is the influence of replacing stiffness of a non-homogeneous material on the absolute value of the basic and higher frequency $\omega_{i}$ depending on material properties and thickness of the facing and core. The next step in vibration analysis of circular plates will be a numerical (MES) and analytical investigation of the influence of a functionally graded material on axisymmetric frequency of Kirchoff's and Reisner-Mindlin's plates with mixed boundary conditions.

\section{Nomenclature}

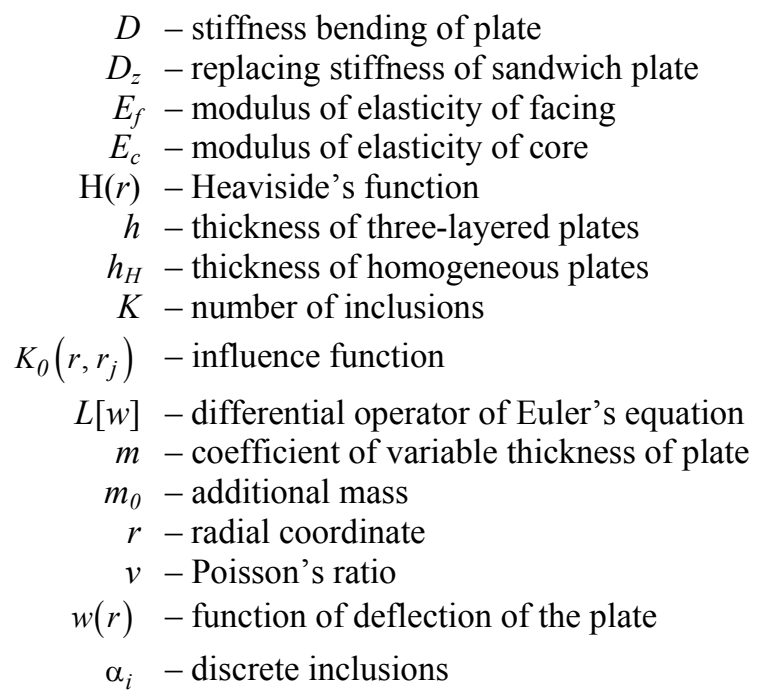


$\delta \quad-$ Dirac's delta

$\rho_{c} \quad$ - density of core

$\rho_{f}-$ density of facing

$\chi_{i, j}-$ coefficients of influence matrix

$\omega_{0}-$ natural basic frequency

\section{References}

Bernstein S.A. and Kieropian K.K. (1960): Opredelenije castot kolebanij sterznevych system metodom spektralnoifunkcii. - Gosstroiizdat.

Conway H. (1958b): Some special solutions for the flexural vibrations of discs of varying thickness. - Engineer. Archives, vol.26, pp.408-410.

Duan W., Quek S. and Wang Q. (2005): Free vibration analysis of piezoelectric coupled thin and thick annular plate.Journal of Sound and Vibration, vol.281, pp.119-139.

Ebrahimi F. and Rastgo A. (2008): An analytical study on the free vibration of smart circular thin FGM plate based on classical plate theory. - Thin-walled Structures, vol.46, pp.1402-1408.

Jaroszewicz J. and Zoryj L.M. (2005): Metody analizy drgań osiowosymetrycznych plyt kolowych z zastosowaniem funkcji wplywu Cauchy'ego. - Technical University of Bialystok Press, 54.

Jaroszewicz J., Żur K.K. and Dragun Ł. (2014): The influence function in analysis of bending curve and reactions of elastic supports of beam with variable distribution of parameters. - Journal of Theoretical and Applied Mechanics, vol.1, pp.247-255.

Kączkowski Z. (2000): Plates: Static Calculations. - Arkady.

Kukla S. and Szewczyk M. (2007): Free vibration of annular plates of stepped thickness resting on Winkler elastic foundation. - Scientific Research of the Institute of Mathematics and Computer Science, vol.2, pp.110-116.

Lal R. and Rani R. (2013): Mode shapes and frequencies of radially symmetric vibrations of annular sandwich plates of variable thickness. - Acta Mechanica, vol.1, pp.1-16.

Magnucki K., Jasion P., Magnucka-Blandzi E. and Wasilewicz P. (2014): Theoretical and experimental study of sandwich circular plate under pure bending. - Thin-Walled Structures, vol.79, pp.1-7.

Roberson R. (1951): Vibration of clamped circular plate carrying concentrated mass. - Journal of Applied Mechanics, vol.18, pp.347-349.

Solecki R. and Szymkiewicz J. (1964): Frame and Plates Systems: Dynamic Analysis. - Arkady.

Sokołowski M. (1958): The bending of transversally non-homogeneous plates of moderate thickness. - Archiwum Mechaniki Stosowanej, vol.10, pp.315-328.

Starovoitov E., Kubenko V. and Tarlakovskii D. (2008): Vibration of circular sandwich plates connected with an elastic foundation. - Structural Mechanics and Strength of Flight Vehicles, vol.52, No.2, pp.151-157.

Szewczyk M, Kukla S. and Zamojska I. (2007): Free vibration of a system of simply supported circular plates connected by elastic elements. - Engineering Modelling, vol.33, pp.153-158.

Timoshenko S. and Woinowsky-Krieger S. (1940): Theory of Plates and Shells. - McGraw-Hill.

Vasylenko N.V. and Oleksiejčuk O.M. (2004): Teoriya kolyvań i stijkosti ruchu. - Vyshcha Shkola.

Zangh L., Hebert R. and Wright J. (2013): Dynamic response of corrugated sandwich steel plates with graded cores. International Journal of Impact Engineering, vol.65, pp.195-194.

Received: July 16, 2014

Revised: January 16, 2015 